\title{
Simulation study of solar wind push on a charged wire: basis of solar wind electric sail propulsion
}

\author{
P. Janhunen ${ }^{1,2}$ and A. Sandroos ${ }^{2}$ \\ ${ }^{1}$ University of Helsinki, Department of Physical Sciences, Finland \\ ${ }^{2}$ Finnish Meteorological Institute, Space Research, Finland
}

Received: 11 May 2006 - Revised: 20 February 2007 - Accepted: 1 March 2007 - Published: 29 March 2007

\begin{abstract}
One possibility for propellantless propulsion in space is to use the momentum flux of the solar wind. A way to set up a solar wind sail is to have a set of thin long wires which are kept at high positive potential by an onboard electron gun so that the wires repel and deflect incident solar wind protons. The efficiency of this so-called electric sail depends on how large force a given solar wind exerts on a wire segment and how large electron current the wire segment draws from the solar wind plasma when kept at a given potential. We use 1-D and 2-D electrostatic plasma simulations to calculate the force and present a semitheoretical formula which captures the simulation results. We find that under average solar wind conditions at $1 \mathrm{AU}$ the force per unit length is $(5 \pm 1) \times 10^{-8} \mathrm{~N} / \mathrm{m}$ for $15 \mathrm{kV}$ potential and that the electron current is accurately given by the well-known orbital motion limited (OML) theory cylindrical Langmuir probe formula. Although the force may appear small, an analysis shows that because of the very low weight of a thin wire per unit length, quite high final speeds (over $50 \mathrm{~km} / \mathrm{s}$ ) could be achieved by an electric sailing spacecraft using today's flight-proved components. It is possible that artificial electron heating of the plasma in the interaction region could increase the propulsive effect even further.
\end{abstract}

Keywords. General or miscellaneous (Instruments useful in three or more fields; New fields (not classifiable under other headings); Techniques applicable in three or more fields)

\section{Introduction}

The level of used propulsion technology is the decisive factor which ultimately sets the scale and scope of human space activity. Besides traditional chemical rockets which can be used both inside and outside of planetary atmospheres,

Correspondence to: P. Janhunen

(pekka.janhunen@fmi.fi) space-only propulsion techniques such as electric propulsion (ion and plasma engines) and solar sails have gained popularity in recent years. Both mentioned techniques have been in principle known for a long time. Likewise, there is much research going on in terms of really advanced (and at the same time long-term) propulsion concepts using e.g. fusion reactors, laser sails or antimatter, with the ultimate goal of enabling interstellar travel.

In this paper we consider a near-term propellantless space physics based sailing method which uses the solar wind momentum flux, i.e. the solar wind dynamic pressure $P_{\mathrm{dyn}}=\rho v^{2}$ which is on the average about $2 \mathrm{nPa}$ at $1 \mathrm{AU}$ distance from the Sun. The idea of using solar wind momentum was first considered by Zubrin and Andrews (1991) who suggested creating an artificial magnetosphere around the spacecraft to deflect the solar wind and thus extract momentum from it. As an alternative way of tapping solar wind momentum the electric sail was suggested by Janhunen (2004). The electric sail consists of a number of long and thin conductive wires or tethers which are kept at a positive potential with the help of an onboard electron gun (one could also consider a negative ion gun, this variant is not pursued in this paper however). Investigating the interaction of the solar wind around a single charged wire and predicting the obtained thrust in different solar wind conditions and different wire potentials is the task of the present paper.

\section{Theory}

Consider a long positively charged wire placed in solar wind which blows perpendicular to it to the x-direction. To calculate the force acting on the wire, it is sufficient to know the potential pattern because from that one can easily compute the solar wind proton trajectories numerically. For each incident proton its deflection angle tells how much $\mathrm{x}$-directed momentum the particle has lost in its interaction with the

Published by Copernicus GmbH on behalf of the European Geosciences Union. 
potential pattern. Only solar wind provides a source of plasma particles, photoelectrons (energies generally below $10 \mathrm{eV}$ ) emitted from the wire are able to move only $\sim 0.1 \mu \mathrm{m}$ away from the wire because of the high surface electric field $\sim 100 \mathrm{MV} / \mathrm{m}$ which tends to pull them back.

In vacuum the potential pattern around a charged wire would be

$V(r)=V_{0} \frac{\ln \left(r_{0} / r\right)}{\ln \left(r_{0} / r_{w}\right)}$

where $r_{0}$ is the distance where the potential is required to vanish $\left[V\left(r_{0}\right)=0\right], r_{w}$ is the wire radius and $V_{0}$ is the wire potential. The plasma electrons shield the potential and make the potential vanish faster than the vacuum solution at high distances. We have found and will show below in detail that the following shielded potential expression ansatz agrees very well with our self-consistent plasma simulation results:

$V(r)=V_{0} \frac{\ln \left[1+\left(r_{0} / r\right)^{2}\right]}{\ln \left[1+\left(r_{0} / r_{w}\right)^{2}\right]}$

where

$r_{0}=2 \lambda_{D e}=2 \sqrt{\frac{\epsilon_{0} T_{e}}{n_{0} e^{2}}}$

where $\lambda_{D e}$ is electron Debye length, $T_{e}$ is the solar wind electron temperature (on average $T_{e}=12 \mathrm{eV}$ at $1 \mathrm{AU}$ ) and $n_{0}$ is the undisturbed solar wind electron density $n_{0}\left(n_{0}=7.3 \mathrm{~cm}^{-3}\right.$ on average at $1 \mathrm{AU})$. Since $r_{w} \ll r_{0}$ it also holds that

$V(r)=\frac{V_{0}}{2} \frac{\ln \left[1+\left(r_{0} / r\right)^{2}\right]}{\ln \left(r_{0} / r_{w}\right)}$.

For $r \ll r_{0}$ potential (2) reduces to the vacuum formula (1) and for $r \gg r_{0}$ it goes to zero as $1 / r^{2}$.

From Gauß' law we find the electron density corresponding to potential (2) as

$$
\begin{aligned}
n_{e}(r) & =n_{0}+\frac{\epsilon_{0}}{e} \frac{1}{r} \frac{d}{d r}\left(r V^{\prime}(r)\right) \\
& =n_{0}\left[1+\frac{e V_{0}}{2 T_{e}} \frac{1}{\left(1+\left(r / r_{0}\right)^{2}\right)^{2} \ln \left(r_{0} / r_{w}\right)}\right] .
\end{aligned}
$$

For $r \ll r_{0}$ the electron density is a constant which is typically 40-60 times higher than $n_{0}$. For $r \gg r_{0}$ its difference to $n_{0}$ goes to zero as $1 / r^{4}$.

The force per unit length acting on the wire is the solar wind dynamic pressure $P_{\mathrm{dyn}}=m_{p} n_{0} v^{2}$ times the effective width of the potential structure. The effective width is proportional to the proton stopping distance $r_{s}$ which must be solved from the equation

$e V\left(r_{s}\right)=(1 / 2) m_{p} v^{2}$.

Thus

$\frac{d F}{d z}=K m_{p} n_{0} v^{2} r_{s} \quad$ with $\quad K \approx 3.09$.
We found the given numerical value for $K$ from a testparticle proton Monte Carlo simulation with potential (2); in other words we launched a number of solar wind protons into the potential pattern and recorded their momentum change after they had finished their interaction with the potential. Solving the stopping distance from Eq. (5) we obtain

$r_{s}=\frac{r_{0}}{\sqrt{\exp \left[\frac{m_{p} v^{2}}{e V_{0}} \ln \left(r_{0} / r_{w}\right)\right]-1}}$,

hence the force per unit length is finally given by

$$
\frac{d F}{d z}=\frac{K m_{p} n_{0} v^{2} r_{0}}{\sqrt{\exp \left[\frac{m_{p} v^{2}}{e V_{0}} \ln \left(r_{0} / r_{w}\right)\right]-1}}
$$

where $r_{0}$ is given by Eq. (3). In most of the interesting range the argument of the exponential in Eq. (8) is around unity so asymptotic formulae for Eq. (8) are not so useful to give.

Figure 1 shows the force per unit length given by Eq. (8) as function of applied voltage $V_{0}$ for three different electron temperatures. The Debye length and thus $r_{0}$, and approximately also $r_{s}$ and $d F / d z$, are proportional to $\sqrt{T_{e}}$, thus higher electron temperature yields wider electron sheath and thus larger propulsive effect.

The bulk of this paper is devoted to providing simulation evidence for the hypothesis that potential (2) is a good approximation for the true potential.

In practise the wire cannot be a single filament because micrometeors would soon break it, but it must instead consist of more than one subwires which are attached together at regular intervals. Ways of constructing such micrometeor resistant wires are known, for example various types of the "Hoytether" wire (Hoyt and Forward, 2001). The perpendicular distance $b$ between the subwires would in practise probably lie in the millimetre to centimetre range; in any case, it satisfies $r_{w} \ll b \ll r_{0}$ where $r_{w}$ is the subwire radius as before (we usually assume $r_{w}=10 \mu \mathrm{m}$ ). In Appendix A we consider how to calculate the effective electric radius of such a multiple wire.

\subsection{Electron current}

The electron current collected by the wire is important to know because it determines the current that the onboard electron gun has to produce in order to maintain the wanted positive potential of the wires. In a stationary state electrons which are trapped by the potential structure of the wire move without colliding with the wire (otherwise the situation would not be stationary), thus only new electrons coming from the surrounding solar wind plasma contribute to the electron current. The orbits of these electrons depend only on the potential structure since the system is collisionless in a very good approximation. Assume first that there is no solar wind proton flow so that the situation is cylindrically 
symmetric. Then the Coulomb force field acting on an incoming electron is a central force field and hence the angular momentum of the particle is conserved, as is its total energy. With these invariants it is easy to calculate how accurately an incoming electron must be "aimed" in order to be in collision course with the wire. The result turns out to be independent of the functional form of the potential structure; it depends only on the wire potential $V_{0}$. The derivation is part of the well-known orbital motion limited (OML) Langmuir probe theory for cylindrical probes (Mott-Smith and Langmuir, 1926; Allen, 1992) and the resulting current per unit length of the cylindrical wire is

$\frac{d I}{d z}=e n_{0} \sqrt{\frac{2 e V_{0}}{m_{e}}} 2 r_{w}$.

We have assumed $e V_{0} \gg T_{e}$ which is very well satisfied since $V_{0}$ is typically $10-20 \mathrm{kV}$ and $T_{e} \approx 12 \mathrm{eV}$. The current does not depend on the electron thermal velocity or the bulk velocity as long as they are much smaller than $\sqrt{e V_{0} / m_{e}}$. To be selfcontained we provide a derivation of Eq. (9) in Appendix B.

We find that our 1-D cylindrically symmetric particle simulation described in Sect. 3 reproduces (9) very accurately. Then, relaxing the cylindrical symmetry assumption, we have also found using a single-particle vacuum Monte Carlo calculation that if there are several parallel wires in general position, the gathered current is just a sum of the single-wire currents given by Eq. (9). Thus, cylindrical symmetry is not essential for Eq. (9) to remain valid. These considerations almost prove that Eq. (9) is the correct law that describes a real electric sail also. A possible source of deviation that we haven't explicitly studied is the deformation of the wire potential structure due to the incident proton flow. Our 2-D simulations reported below show, however, that this deformation is small near the wire (despite the fact that the deformation is the essential mechanism by which the solar wind pushes the wire). Furthermore, since cylindrical symmetry and electron bulk speed are both inessential for Eq. (9) to remain valid, we have high confidence that it remains valid also in the completely general case.

\section{1-D simulation}

Our first electrostatic simulation has cylindrical symmetry and includes only electrons with undisturbed density $n_{0}$. Ions are assumed to form a neutralising constant background whose density is everywhere equal to $n_{0}$. The wire with radius $r_{\text {min }}$ is located at the centre. It absorbs all electrons colliding it and contains a given positive line charge. The source line charge is an arbitrary user-specified function of time. The cylindrically symmetric geometry allows us to use Gauß' law for calculating the electric field at each particle by counting the number of electrons inside the radius. This approach has the benefit of not requiring a spatial grid so that the only numerical parameters whose effect must be studied

\section{Force per unit length}

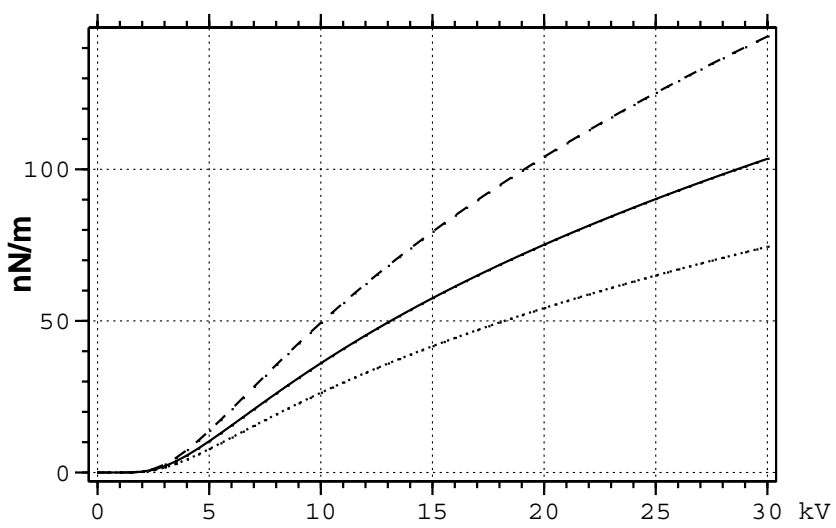

Fig. 1. Force per unit length according to Eq. (8) as function of voltage $V_{0}$. Solid curve is for baseline electron temperature $12 \mathrm{eV}$, dotted for $6 \mathrm{eV}$ and dashed for $24 \mathrm{eV}$. Solar wind electron density $n_{0}=7.3 \mathrm{~cm}^{-3}$, velocity $v=400 \mathrm{~km} / \mathrm{s}$ and wire radius $r_{w}=10 \mu \mathrm{m}$.

are the number of particles and the timestep (Birdsall and Langdon, 1991). The particle list must be kept sorted in the radial coordinate. Angular momentum conservation is built into the equations analytically. The equations of motion are

$$
\frac{d v_{r}}{d t}=a_{r}+\frac{L^{2}}{r^{3}}, \quad \frac{d r}{d t}=v_{r}
$$

where $v_{r}$ is the radial speed, $L$ the angular momentum divided by electron mass and $a_{r}=\left(-e / m_{e}\right) E_{r}$ is the acceleration due to Coulomb force. This gridless approach is not necessarily the most economical to use, but computing speed is not our main aim in this 1-D simulation which runs rather fast in any case. Our complementary 2-D simulation described in Sect. 4 does use a grid.

Figure 2 shows the 1-D plasma simulation results in saturated state. Shown are the radial profile of the electron density together with the analytic model expression (4). One sees that the agreement is very good except near the wire where the wire absorbs electrons which is not taken into account by the analytic model. For practical reasons our wire radius in the 1-D simulation $r_{\mathrm{min}}=1 \mathrm{~m}$ which is much larger than in reality. Using a smaller value would be possible, and we have also tested lower values, but then only few electrons exist near the wire which causes fluctuations in the results. Since our main aim is to verify the working of the analytic theory presented above, using realistic value for $r_{\min }$ is not essential.

Figure 2 also shows the simulated radial potential profile together with Eq. (2). The vacuum potential, Eq. (1), is also shown for comparison by dotted line. Again we see that Eq. (2) is a very good approximation for the simulation results. The main difference occurs toward the outer edge of the simulation cylinder at $r_{\max }=50 \mathrm{~m}$ where the simulation 


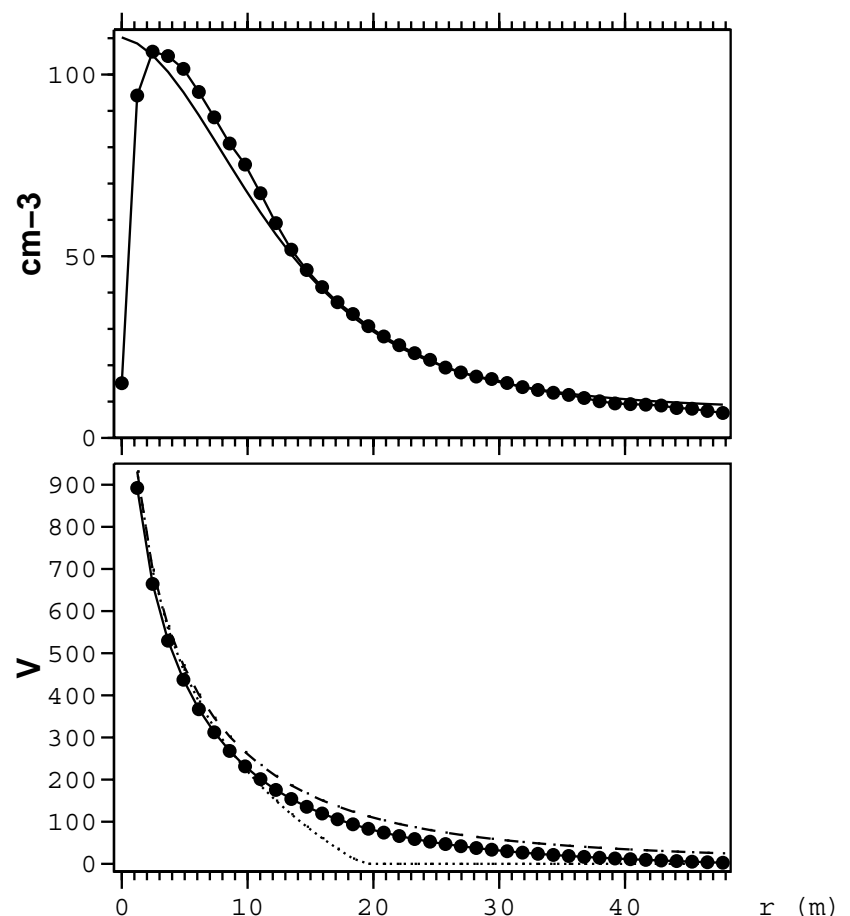

Fig. 2. 1-D plasma simulation results. Upper panel: Radial profile of electron density from simulation (dot-marked line), model density Eq. (4) (solid line). Lower panel: Simulated potential (dotmarked line), model potential Eq. (2) (dashed line), vacuum potential Eq. (1) (dotted line). Negative values of the vacuum potential have been replaced with zero.

potential is forced to vanish by our Dirichlet boundary condition while Eq. (2) goes to zero only asymptotically as $r \rightarrow \infty$. Renormalising Eq. (2) so that $V\left(r_{\max }\right)=0$ would make the model and simulation curves nearly indistinguishable.

\section{2-D simulation}

In this section we describe 2-D electrostatic particle-in-cell (PIC) simulations. The simulation plane (xy-plane) is perpendicular to the wire and the solar wind moves along positive $x$. A spatial grid is used as is customary in 2-D simulations (Birdsall and Langdon, 1991).

We use a grid spacing $\Delta x=1.25 \mathrm{~m}$ in $x$ and $y$ and the box size is $L_{x}=320 \mathrm{~m}$ in $x$ and $L_{y}=160 \mathrm{~m}$ in $y$ so that the spatial grid is of size $256 \times 128$. We simulate only the northern semicylinder $(y>0)$ and use a mirroring particle boundary condition and vanishing potential $y$-derivative at $y=0$ (Neumann condition). The potential $\Phi$ is set to zero at $x= \pm L_{x} / 2$ (Dirichlet condition). On the top boundary $\left(y=L_{y}\right), \partial \Phi / \partial y=0$ is assumed (i.e. Neumann condition). The number of electrons per grid cell is 20 in undisturbed solar wind. The timestep is $15.625 \mathrm{~ns}$. Most runs were continued at least for $40 \mathrm{~ms}$ while some were extended to $100 \mathrm{~ms}$.
The risetime of the potential from zero to final value was set to $5 \mathrm{~ms}$. Different risetimes produced no difference in the saturated state.

In this simulation we introduce the wire potential so that the vacuum electric field of the wire is added to the particle force in analytic form each time a particle is moved. Within a radius of $\Delta x$ from the wire position $(x, y)=(0,0)$ the wire electric field magnitude is modified so that the field is proportional to the radial coordinate in this region. This method of regularising the wire electric field corresponds to the situation where the wire is effectively a uniformly charged cylinder with radius equal to $\Delta x$. In contrast to the 1-D simulation described above, no particle absorption is done in this region. This corresponds to reality because the actual wire is so thin that the probability of an electron hitting it would be practically zero in the scale of this 2-D simulation. We use the 2-D simulation only to evaluate the force acting on the wire and do not attempt to calculate the electron current independently any more.

The self-consistent part of the electric field is performed in traditional PIC manner, using area weighting in charge assignment, linear interpolation in the electric field and ninepoint stencil in evaluating the potential gradient (Birdsall and Langdon, 1991).

We also include solar wind helium ions (alpha particles) in the simulation. The effect of considering helium has only a minor effect on the propulsive effect, however. Having two times smaller $q / m$ ratio than protons, the alpha particles penetrate more efficiently into the potential structure causing a smaller propulsive effect per unit mass than protons. If one does not want to include helium ions, we found that one obtains a quite accurate result by using the solar wind electron density and assuming that all ions are protons.

Figure 3 shows the saturated state of the 2-D PIC simulation in our baseline run. The ion density (top panel) clearly shows how incoming ions are repelled by the wire potential and an ion deflection pattern develops, the shape of which much resembles the familiar magnetosphere-solar wind interaction. The middle and bottom panels show the total and plasma-only potentials, respectively. These are remarkably symmetrical in $x$, i.e. the potential in the region where protons are most efficiently deflected is nearly cylindrically symmetric. Thus the calculations of Sects. 2 and 3 remain well valid also when the solar wind is introduced, although, it must be remarked, a small deviation from symmetry is the very agent which produces the force acting on the wire.

One-dimensional potential profiles along the subsolar line (x-axis) as taken from the 2-D simulation are shown in Fig. 4. The vacuum potential of the wire (Eq. 1, dashed) looks linear in this plot where the $\mathrm{x}$-axis is logarithmic. The plasma potential (curve marked with heavy dots) varies smoothly. Both $x>0$ and $x<0$ branches are plotted in the figure, but they virtually overlap in this scale, which is another manifestation of said high degree of cylindrical symmetry. The remaining curve shows the total potential together with Eq. (2) 


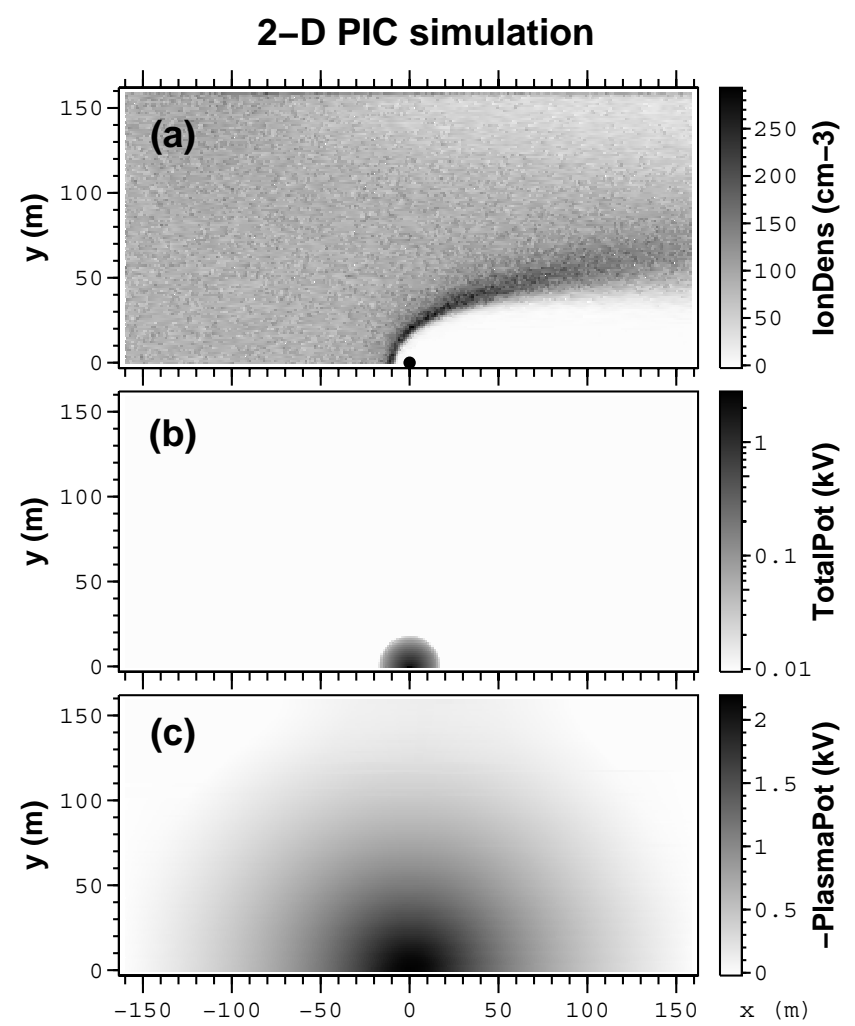

Fig. 3. Saturated state (at $t=20 \mathrm{~ms}$ ) of the baseline 2-D PIC simulation. Instantaneous ion density (a), potential (b) and negative of the part of the potential which is due to the plasma (c). Scale is logarithmic in (b) and linear in (a) and (c). Solar wind arrives from the left and the wire is at $(x, y)=(0,0)$. Panels (b) and (c) have been time averaged over $0.5 \mathrm{~ms}$.

optimally fitted to it (including an offset). The fitted form is again nearly indistinguishable from the simulation curve in this scale. Thus the analytic theory of Sect. 2 is a good approximation also to the 2-D simulation results.

We calculate the force per unit length $d F / d z$ by two complementary methods from the 2-D simulation. In the first method (method 1) we evaluate the Coulomb force $\mathbf{F}=q \mathbf{E}$ where $q$ is the wire charge and $\mathbf{E}$ is the electric field at the wire. In the second method (method 2) we record the timeaveraged electric field pattern from the simulation and as a post-processing step compute the $\mathrm{x}$-directed momentum budget of test ions moving in the obtained electric field. We also tested a force calculation method (method 3$)$ where the momentum budget of the simulation particles is considered directly. Method 3 (not shown) gives consistently less accurate results than the other methods probably because of fluctuations in the fields which are due to the finite number of particles. These fluctuations are efficiently suppressed by the time-averaging step involved in method 2.

In Table 1 we show the effect of various numerical parameters on the obtained force per unit length using both methods 1 and 2. Reducing the timestep from 31.25 to $15.625 \mathrm{~ns}$

\section{2-D PIC simulation potentials}

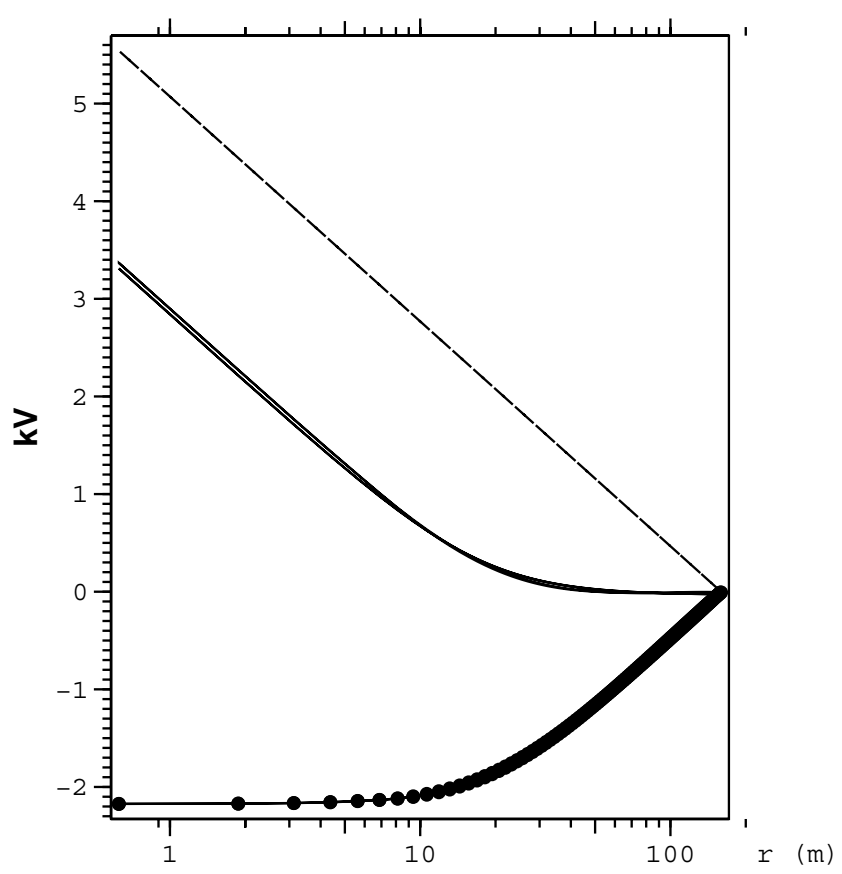

Fig. 4. One-dimensional potential profiles along subsolar line taken from the 2-D PIC simulation at $t=20 \mathrm{~ms}$. Dashed line (topmost curve) is the source potential profile of the wire. Line marked with dots (bottommost curve) is the plasma potential from the simulation. Solid line in the middle is actually two lines very close together, one is the total potential (plasma plus source) and the other is the function $\left.\left(V_{0} / 2\right) \ln \left(1+\left(r_{0} / r\right)^{2}\right) / \ln \left(r_{0} / r_{w}\right)\right)+C$ (Eq. 2) with $r_{w}=10 \mu \mathrm{m}, r_{0}=19.97 \mathrm{~m}$ and $C=-10.68 \mathrm{~V}$ fitted to the first one. Notice logarithmic scale in horizontal axis.

Table 1. Effect of grid spacing $\Delta x$, box length $L_{x}$, timestep $\Delta t$ and number of ions per cell in solar wind $N_{\text {cell }}$ on Method 1 and 2 for per unit length $d F_{1} / d z$ and $F_{2} / d z$, respectively. Last line corresponds to the standard run.

\begin{tabular}{llllll}
\hline$\Delta x$ & $L_{x}$ & $\Delta t$ & $N_{\text {cell }}$ & $d F_{1} / d z$ & $d F_{2} / d z$ \\
\hline $1.25 \mathrm{~m}$ & $160 \mathrm{~m}$ & $31.25 \mathrm{~ns}$ & 10 & $56 \mathrm{nN} / \mathrm{m}$ & $57 \mathrm{nN} / \mathrm{m}$ \\
$1.25 \mathrm{~m}$ & $160 \mathrm{~m}$ & $15.625 \mathrm{~ns}$ & 10 & $41 \mathrm{nN} / \mathrm{m}$ & $45 \mathrm{nN} / \mathrm{m}$ \\
$1.25 \mathrm{~m}$ & $160 \mathrm{~m}$ & $7.8125 \mathrm{~ns}$ & 10 & $42 \mathrm{nN} / \mathrm{m}$ & $44 \mathrm{nN} / \mathrm{m}$ \\
$0.625 \mathrm{~m}$ & $160 \mathrm{~m}$ & $15.625 \mathrm{~ns}$ & 10 & $44 \mathrm{nN} / \mathrm{m}$ & $48 \mathrm{nN} / \mathrm{m}$ \\
$1.25 \mathrm{~m}$ & $160 \mathrm{~m}$ & $15.625 \mathrm{~ns}$ & 5 & $40 \mathrm{nN} / \mathrm{m}$ & $41 \mathrm{nN} / \mathrm{m}$ \\
$1.25 \mathrm{~m}$ & $320 \mathrm{~m}$ & $15.625 \mathrm{~ns}$ & 20 & $48 \mathrm{nN} / \mathrm{m}$ & $53 \mathrm{nN} / \mathrm{m}$ \\
\hline
\end{tabular}

and keeping the box length at $160 \mathrm{~m}$ and grid spacing $1.25 \mathrm{~m}$ reduces the force by $\sim 25 \%$, but further reduction of the timestep brings no further change. Keeping the timestep at the reduced value $15.625 \mathrm{~ns}$ and reducing also the grid spacing to $0.625 \mathrm{~m}$ the force slightly increases. If one makes the simulation more inaccurate by reducing the number of 


\section{Force per length from 2-D PIC}

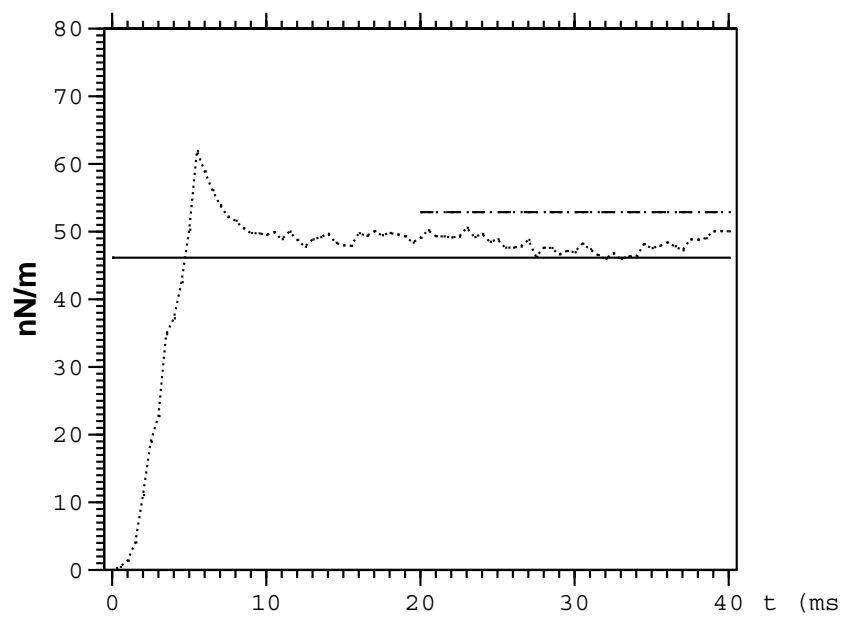

Fig. 5. Force per unit length from 2-D PIC simulation as a function of time using the Coulomb force method (method 1, dotted) and the particle momentum method averaged over 20-40 ms (method 2, dashed). The risetime of the wire potential was set to $5 \mathrm{~ms}$. The solid line shows the model result from Eq. (8).

particles per grid cell from 10 to 5, the force gets slightly reduced. In the baseline run (last line of Table 1) we thus use 20 ions per cell in the undisturbed solar wind to ensure that their number is sufficient and use a box length of $320 \mathrm{~m}$ to have some additional safety margin in terms of the domain size as well. This safety margin is good to have when we next start varying the solar wind parametres and the electron sheath (potential structure) size starts to vary as well.

The force by methods 1 and 2 from the baseline run is shown in Fig. 5 as a function of time. The expected result from Eq. (8) is shown by solid line and the method 2 result, averaged over $20-40 \mathrm{~ms}$, is shown by a dashed line. In this case, with all methods the simulation-based force is somewhat larger than the theoretical formula. The data points in other figures show time averaged quantities where the averaging is carried out from 20 to $40 \mathrm{~ms}$, thus the initial transients at $\sim 6 \mathrm{~ms}$ do not contribute to the results.

The potential in Figs. 5, 7, 8 and 9 is about $14.5 \mathrm{kV}$. The total potential in the simulation box is a sum of the bare wire potential which is imposed and known and the plasma potential which is calculated during the simulation. The potential difference $V_{0}$ between the wire surface and the solar wind plasma must therefore be calculated from the simulation afterwards. The $V_{0}$ values thus obtained differ slightly from case to case. Although not numerically significant, we compensate for the differences by scaling with Eq. (8) in Figs. 7, 8 and 9.

Figure 6 shows the simulated force as a function of the potential $V_{0}$. Again, method 1 (triangles) and method 2 (dots) are compared with the theoretical curve, Eq. (8). In addition

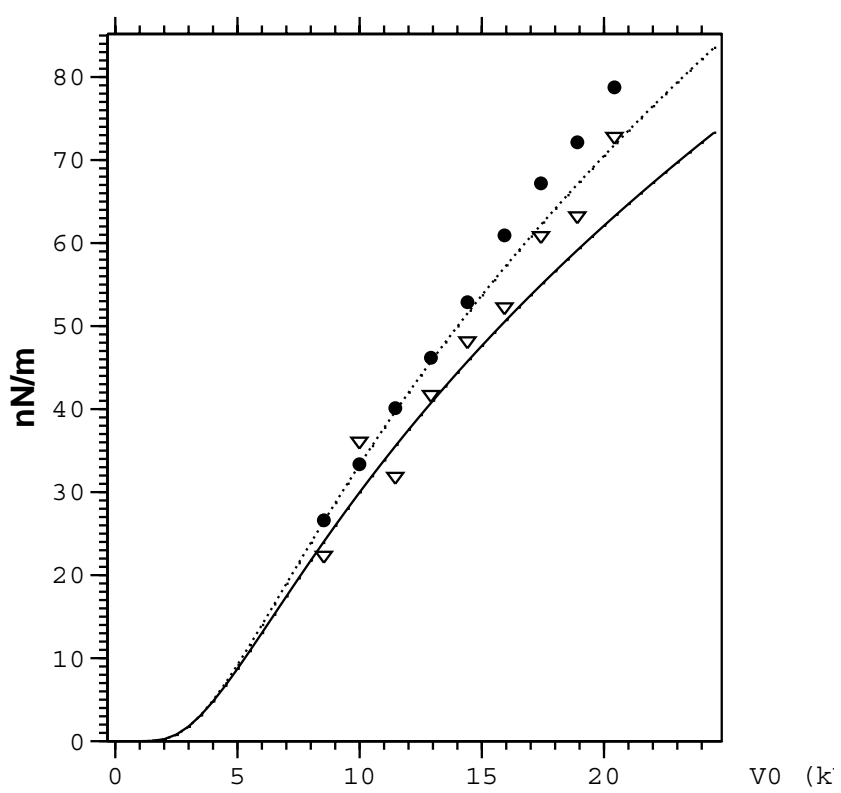

Fig. 6. Force per unit length from 2-D PIC simulation as a function of wire voltage $V_{0}$ compared with model prediction. Force calculated from electric field at wire (method 1, triangles), from particle momentum balance (method 2, dots), force from and Eq. (8) (solid) and force from Eq (8) multiplied by 0.6 and $T_{e}$ replaced by $\sqrt{T_{e}^{2}+(30 \mathrm{eV})^{2}}$ (dotted). Motivation for dotted line expression is given in connection with Fig. 9. In each case the risetime was $5 \mathrm{~ms}$, the run was continued until $40 \mathrm{~ms}$ and the forces were averaged at $20-40 \mathrm{~ms}$.

we show a version of Eq. (8) which is multiplied by 0.6 and where $T_{e}$ is replaced by $\sqrt{T_{e}^{2}+(30 \mathrm{eV})^{2}}$ by dotted line; the motivation for this is given in connection with Fig. 9 below. One sees that the agreement between the theoretical curves and the simulation (both methods 1 and 2) is satisfactorily good.

Figure 7 is similar to Fig. 6 except that now we vary the solar wind electron density $n_{0}$ instead of the voltage. The agreement between theory, Eq. (8), and simulation is good for low and normal density and becomes worse for higher densities. This is probably due to the fact that for high electron density, the size of the electron sheath (potential structure) is smaller so that one should use a finer grid to resolve it properly.

In Fig. 8 we vary the solar wind speed. Now the optimal force position occurs at somewhat different solar wind speed in the theory and the simulation. Again, the discrepancy between methods 1 and 2 is largest at the highest solar wind speed, which is probably again due to the fact that the size of the electron sheath is smaller and more difficult for the simulation to resolve.

Finally, in Fig. 9 we vary the solar wind electron temperature $T_{e}$. All methods predict that the force increases when the electrons become hotter. The simulation and theory do 


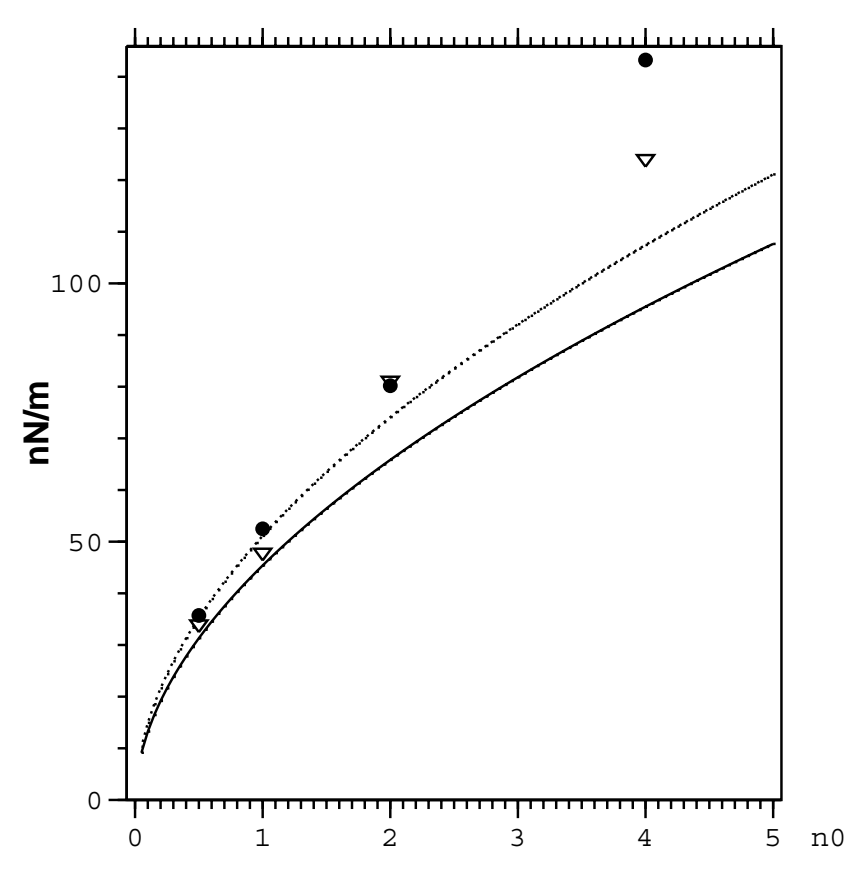

Fig. 7. Same as Fig. 6, but as function of solar wind electron density, normalised to $7.3 \times 10^{6} \mathrm{~m}^{-3}$.

not quite agree upon the functional form, however. For high $T_{e}$ the simulation predicts about $60 \%$ of the value predicted by Eq. (8), while for small $T_{e}$ the simulated force does not approach zero but tends to a finite value. It is possible that for small $T_{e}$, numerical electron heating, due to the stochastic nature of the simulation and the discreteness of the grid (Birdsall and Langdon, 1991), is significant in comparison with the enforced $T_{e}$. To illustrate this qualitatively, the dotted line in Figs. 6-9 is computed from a version of Eq. (8) where $T_{e}$ is replaced by $\sqrt{T_{e}^{2}+(30 \mathrm{eV})^{2}}$ and the result is multiplied by 0.6 . This form has been selected to give a satisfactory fit to the simulation results in Fig. 9. If this numerical electron heating interpretation is correct, one should insert a correction factor of $\sim 0.6$ in front of Eq. (8). On the other hand, numerical heating would be expected to secularly increase $T_{e}$ and thus $d F / d z$ throughout the run, while the simulated force actually stays constant in time (Fig. 5). Thus the question of the true magnitude of the force remains open, the factor 0.6 however gives an indication of the maximal magnitude of the uncertainties involved.

All in all, we conclude that the 2-D simulation results are in good agreement with each other and the theory, Eq. (8). The total time thus far consumed by the runs is about 20000 CPU hours (2.3 CPU years), corresponding to $\sim 10^{14}$ particle updates. This includes both the runs documented here and also some other runs that we have performed with different timestep, number of particles, grid spacing and box size. The employed code is a descendant of the code used by Janhunen et al. (2003). In the initial stage of our work we also used the general-purpose particle code XOOPIC (Verboncoeur et al.,

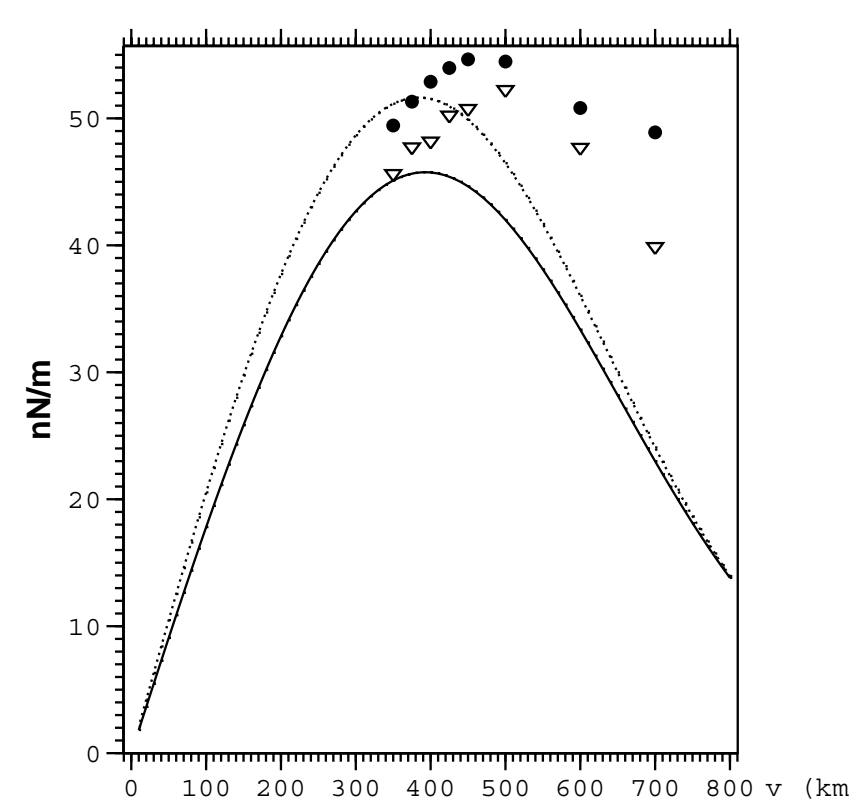

Fig. 8. Same as Fig. 6, but as function of solar wind speed.

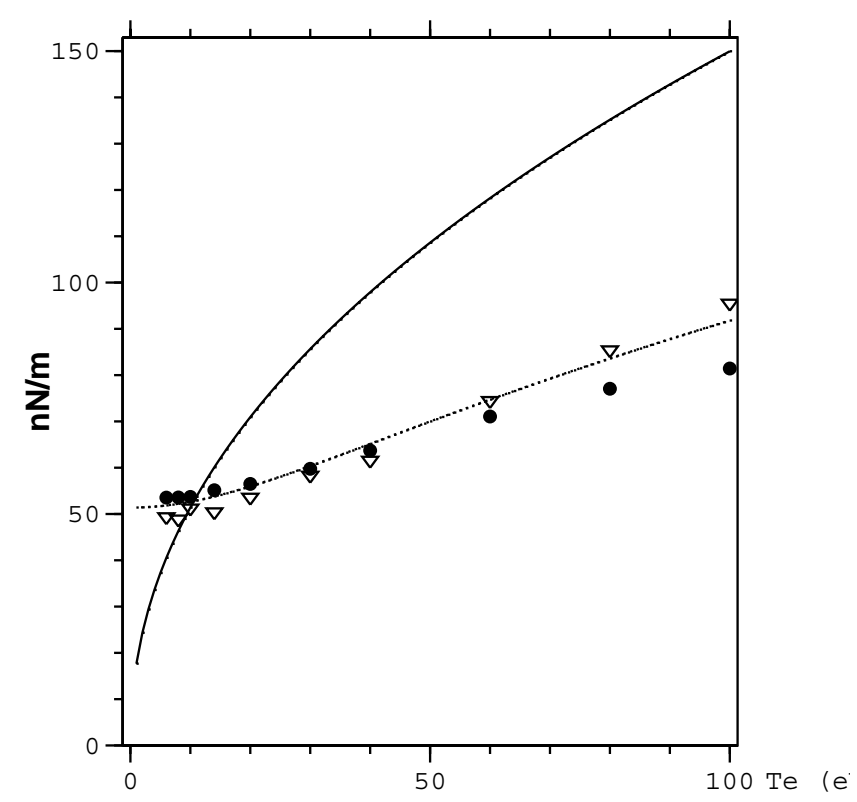

Fig. 9. Same as Fig. 6, but as function of solar wind electron temperature. Again, the dotted line is a version of Eq. (8) where $T_{e}$ was replaced by $\sqrt{T_{e}^{2}+(30 \mathrm{eV})^{2}}$ and the result multiplied by 0.6 .

1995) to verify that our code and XOOPIC produced similar results in cases with simple enough boundary conditions where a comparison was possible. 


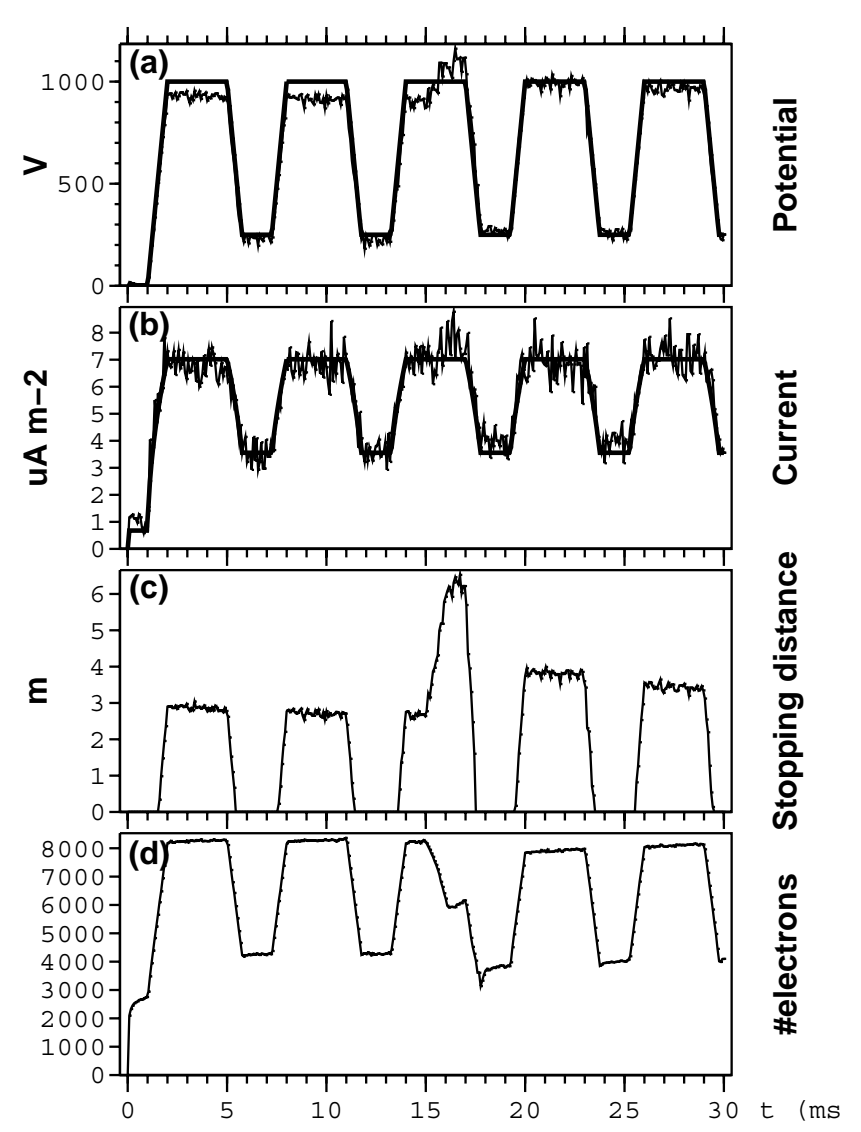

Fig. 10. Results of 1-D simulation with periodic voltage input and artificial electron heating period included at 14.9-16.1 ms. Driver and actual potential (a), model (Eq. 9) and actual current density on wire (b), proton stopping distance $r_{s}$ of formed potential structure (c), number of electrons in simulation box (d). In panels (a) and (b), piecewise linear thick line is analytic model and thin fluctuating curve is actual simulation result.

\section{Possible benefit of electron heating}

The force formula Eq. (8) depends on the solar wind electron temperature $T_{e}$ approximately as $\sqrt{T_{e}}$ : higher temperature produces higher propulsive effect (see also Fig. 9). We cannot change the solar wind electron temperature artificially, but for increasing the propulsive effect it might be actually sufficient to heat only those electrons which are trapped by the wire potential. To test this idea we run the 1-D simulation with artificial electron heating. First we need to explain some other particularities of the run, however.

We use the proton stopping distance $r_{s}$ of Eq. (7) as an easily computable proxy for the propulsive effect. We also run the wire with an input voltage which is piecewise linear and periodic in time (Fig. 10, panel a). The resulting electron current (panel b) also comes out as periodic: the system has no "memory" in this respect. For time $t$ in $14.9 \mathrm{~ms} \leq t \leq 16.1 \mathrm{~ms}$, we insert ad hoc electron heating by multiplying each electron velocity at each timestep by $1+\epsilon$ where $\epsilon=2 \times 10^{-5}$ if the electron is closer than $r_{0}=20 \mathrm{~m}$ to the centre. The timestep is $\Delta t=25 \mathrm{~ns}$, thus the heating corresponds to e-folding time of $2.5 \mathrm{~ms}$. Panel (c) of Fig. 10 shows how the proton stopping distance reacts to the heating: it jumps about twofold during the $1.2 \mathrm{~ms}$ heating period. The effect of the heating is still visible during the fourth voltage maximum at $20-23 \mathrm{~ms}$. The interpretation is that once the trapped population has been heated, the temperature remains high except for those electrons that escape from the potential well while the potential is lower.

It is not easy to study the long-time behaviour reliably with PIC simulations because due to numerical effects, the simulations are not nearly as collisionless as real plasma (Birdsall and Langdon, 1991). In the simulation, collisions cause the small decline of the stopping distance which is visible already during $0-10 \mathrm{~ms}$ in panel (c) of Fig. 10. We have tested that the results remain qualitatively similar regardless of whether heating concerns only the radial or the azimuthal velocity component or if it concerns only those electrons moving in one direction.

One could perhaps implement electron heating by emitting radio wave power to the electron sheath, either from an antenna or perhaps by modulating the electron gun beam at high frequency. For evaluating the feasibility of the approach in terms of power consumption etc., one would need to determine the heating power. The needed heating power depends on how fast the heating has to be, which in turn depends on the electron cooling mechanisms. There are not many effective cooling mechanisms, however: radiative cooling is very slow, as is also cooling due to Coulomb collisions between electrons. (There are no ions in the electron sheath.) The dominant cooling mechanism may be the fact that part of the trapped electron population is lost and replaced by a new one each time the potential of the wire is lowered for some reason, usually some guiding manoeuvre or as response to changed solar wind. Quite possibly such potential changes can be made infrequent enough so that significantly higher than natural electron temperatures can be maintained in the electron sheaths for most of the time, with an associated increased propulsive effect.

To have a rough idea of the required power levels for heating, let us compare it with the power which is needed by the electron gun for compensating the electron current (Eq. 9). From Eq. (4) we find that the electron density near the wire is

$n_{e w}=\frac{n_{0}}{2} \frac{e V_{0}}{T_{e}} \frac{1}{\ln \left(r_{0} / r_{w}\right)}$.

Since this electron density extends roughly until $r_{0}$ (Fig. 2), the number of electrons per unit length of the sheath is approximately

$\frac{d N}{d z}=n_{e w} \pi r_{0}^{2}=\frac{\pi}{2} n_{0} r_{0}^{2} \frac{e V_{0}}{T_{e}} \frac{1}{\ln \left(r_{0} / r_{w}\right)}$.

The electron gun power per unit length of the wire is $d P / d z=V_{0}(d I / d z)$ where $d I / d z$ is given by Eq. (9). 
Assuming that the radio heating power of electrons is a fraction $\eta$ of $d P / d z$ we obtain for the doubling timescale of the electron temperature

$\tau \simeq \frac{T_{e} d N / d z}{\eta V_{0}(d I / d z)}=\frac{\pi}{4} \frac{1}{\eta} \frac{r_{0}^{2}}{r_{w}} \sqrt{\frac{m_{e}}{2 e V_{0}}} \frac{1}{\ln \left(r_{0} / r_{w}\right)}$.

Inserting typical numerical values $r_{0}=20 \mathrm{~m}, r_{w}=10 \mu \mathrm{m}$ and $V_{0}=15 \mathrm{kV}$ we find $\tau \approx 0.03 \mathrm{~s} / \eta$. For example if the radio emission power is $\approx 10 \%$ of the electron gun power and the heating efficiency is $10 \%$ (it cannot be near $100 \%$ as the collisionless skin depth $c / \omega_{p e}$ is $\approx 10$ times larger than the sheath thickness $r_{0}$ ), we have $\eta=0.01$ and $\tau=3 \mathrm{~s}$ for the electron temperature doubling time. The shortness of this timescale compared with typical variations in the solar wind gives us hope that the electron heating approach may be able to boost performance further, although much research remains to be done concerning its details.

\section{Feasibility as propulsive method}

Consider a simplified mission setup where an electric sail spacecraft starts from Sun orbit at $1 \mathrm{AU}$ $\left(1 \mathrm{AU}=1.496 \times 10^{11} \mathrm{~m}\right)$ and accelerates radially outward in the solar system. The competing radial forces affecting the spacecraft trajectory are the outward electric sail thrust and the inward solar gravity. We need to determine how the electric sail thrust depends on the radial distance $r$ from the Sun. The solar wind density $n_{0}$ goes as $1 / r^{2}$ and the speed $v$ stays nearly constant (Kivelson and Russell, 1995) while the electron temperature $T_{e}$ behaves approximately as $r^{-1 / 3}$ (Sittler and Scudder, 1980; Slavin and Holzer, 1981). From Eq. (8) we see that if we ignore the $r_{0}$-dependence inside the logarithm, the thrust $F$ goes as

$F \sim n_{0} r_{0} \sim \sqrt{n_{0} T_{e}} \sim \sqrt{\frac{1}{r^{2}} \frac{1}{r^{1 / 3}}}=\left(\frac{1}{r}\right)^{7 / 6}$.

Solving the equation of motion (Appendix C) gives for the asymptotic speed at far distance

$v_{f}=\sqrt{\left(12 a_{0}-g_{0}\right) R_{0}}$

where $a_{0}$ is the sail acceleration at the starting distance $R_{0}\left(R_{0}=1 \mathrm{AU}\right)$ and $g_{0}$ is solar gravity field at $R_{0}$ $\left(g_{0}=5.93 \mathrm{~mm} / \mathrm{s}^{2}\right)$. For Eq. (15) to be valid the acceleration $a_{0}$ must be high enough that the orbit escapes Sun's gravity field. The condition for this is $a_{0}>g_{0} / 12$. The decrease of the relative solar wind speed with respect to the spacecraft when the spacecraft is moving fast is ignored in this simple analysis.

The acceleration at $1 \mathrm{AU}, a_{0}$, is the sail thrust force divided by the total mass of the spacecraft. To derive a conservative estimate for the acceleration, we use the already flown SMART-1 mission as a starting point. SMART-1 had a $1.19 \mathrm{~kW}$ ion engine onboard and its dry mass was
$287 \mathrm{~kg}$. This dry mass contained everything: the spacecraft bus (frame), solar panels, power conversion systems, the ion engine and scientific instruments. In the electric sailing spacecraft one has an electron gun instead of an ion engine but otherwise the designs could be similar. Electron guns are somewhat simpler than corresponding ion emitters, thus we can take the SMART-1 values as a conservative estimate for the power versus mass ratio of the spacecraft body:

$$
\left(\frac{P}{m}\right)_{\text {body }}=\frac{1190 \mathrm{~W}}{287 \mathrm{~kg}}=4.15 \mathrm{~W} / \mathrm{kg} \text {. }
$$

At $r>1$ AU solar panels produce less power so that the electron gun current gets diminished there, which at first sight appears to be a problem. However, the electron current gathered by the wires is proportional to the solar wind density $n_{0}$ (Eq. 9) and thus it has the same $1 / r^{2}$ radial dependence as Sun's photon field. Thus the same fraction of the available solar panel power is needed at all radial distances from the Sun on the average.

Assume that the wires are $10 \mu \mathrm{m}$ thick and made of material whose density is $4000 \mathrm{~kg} / \mathrm{m}^{3}$ (intermediate between steel and carbon fibre). We calculate everything per unit length of the wire $(d / d z)$. The mass coming from the spacecraft body is

$$
\left(\frac{d m}{d z}\right)_{\text {body }}=\frac{V_{0}(d I / d z)}{(P / m)_{\text {body }}}=4.4 \mathrm{mg} / \mathrm{m}
$$

where $d I / d z$ was given by Eq. (9) and $V_{0}=12 \mathrm{kV}$ was used. Likewise we obtain for the wires

$$
\left(\frac{d m}{d z}\right)_{\text {wires }}=\pi r_{w}^{2} \rho_{w}=1.26 \mathrm{mg} / \mathrm{m} \text {. }
$$

Then we get the acceleration at $1 \mathrm{AU}$ :

$a_{0}=\frac{d F / d z}{(d m / d z)_{\text {body }}+(d m / d z)_{\text {wires }}}=8.4 \mathrm{~mm} / \mathrm{s}^{2}$.

where $d F / d z$ was given by Eq. (8). Using Eq. (15) we obtain $v_{f}=119 \mathrm{~km} / \mathrm{s}$.

The above calculation was without any payload, where by payload we mean everything not directly related to propulsion, including the spacecraft bus. Assuming e.g. equal masses for the electric sail package and the payload, the acceleration gets halved to $4.2 \mathrm{~mm} / \mathrm{s}^{2}$ and for the final speed one now obtains $v_{f}=82 \mathrm{~km} / \mathrm{s}$. For concreteness, assuming total mass of $60 \mathrm{~kg}$ ( $30 \mathrm{~kg}$ sail plus $30 \mathrm{~kg}$ payload) one obtains that the total length of wire in the sail must be $2100 \mathrm{~km}$. Using Eq. (18), the mass of the wires is only $2.6 \mathrm{~kg}$. A technically feasible way to set up the wires seems to be to divide them into a number of independent radial lines which are kept stretched by spacecraft and wire system rotation. In our example one could have e.g. 100 radial wires of $21 \mathrm{~km}$ length each. Near the spacecraft the wires are closer to each other than $r_{0}$ so that their potential structures overlap which has a small effect on performance. An upper limit of $\sim 100 \mathrm{~km}$ is 
set by the material conductivity of the wires (they must be able to carry the arriving electron current) while the number of wires is in practise limited by technical aspects.

With $82 \mathrm{~km} / \mathrm{s}$ speed (17.2 AU/year) one would reach Pluto orbit in two years.

We have made more elaborate calculations of the performance where we among other things use real solar wind data and take into account the wire multiplicity which is needed for micrometeor resistance (Hoyt and Forward, 2001). These considerations typically reduce the final speeds to some extent, but broadly speaking, using different assumptions the resulting final speeds generally fall in the range $40-100 \mathrm{~km} / \mathrm{s}$ for payloads up to $\sim 50 \mathrm{~kg}$, assuming power versus mass ratios that correspond to today's flight-proved components for the spacecraft body.

Here we did not include a correction factor of 0.6 in Eq. (8), whose presence would explain better the electron temperature behaviour (see the second-last paragraph of Sect. 4 and Fig. 9). The final speed has approximately a square root dependence on the acceleration, so with this correction the final speed in the above example would become $60 \mathrm{~km} / \mathrm{s}$ instead of $82 \mathrm{~km} / \mathrm{s}$. On the other hand, we also did not assume any electron heating. If electron heating would be implemented successfully, the performance could be larger, possibly by a significant amount.

The results of this paper should be accurate enough for evaluating the overall feasibility of the electric sail. For detailed mission planning, space experiments with a prototype sail would be needed.

\subsection{Comparison with solar sail}

A comparison of the electric sail performance with other proposed propulsion methods such as electric propulsion is outside the scope of this paper. However, the solar radiation pressure sail is in many respects similar enough to allow for a rather simple quantitative comparison.

An ideal (i.e. fully reflecting) solar sail receives a radiation pressure force of $9 \mu \mathrm{N} / \mathrm{m}^{2}$ at $1 \mathrm{AU}$ distance from the Sun. Let us calculate how thin a solar sail should be, to reach the same specific acceleration as an electric sail wire plus electron gun subsystems (Eq. 19). Using the above example with $82 \mathrm{~km} / \mathrm{s}$ final speed, one obtains that the solar sail should have an areal density of $1.1 \mathrm{~g} / \mathrm{m}^{2}$, which translates to $200 \mathrm{~nm}$ thickness if the material is aluminium and $50 \%$ of the mass is assumed to go to support structures. This is $5-10$ times thinner than present technology.

Although similar in many respects, the solar sail and the electric sail also have some important operational differences. When moving away from the Sun, the electric sail force decays as $1 / r^{7 / 6}$ which is slower than the solar sail $1 / r^{2}$ dependence. On the other hand the solar sail can be used also inside planetary magnetospheres, whereas the electric sail needs the solar wind to operate.

\subsection{Possible missions}

Assuming that no technological obstacles will arise that markedly alter the performance of the electric sail from the above estimates, which type of missions could benefit from it? The electric sail much resembles the solar sail in that it provides small but inexhaustible thrust which is directed outward from the Sun, with a modest control of the thrust direction allowed (probably by a few tens of degrees). First and foremost the electric sail can thus be used for missions going outward in the solar system and aiming for $>50 \mathrm{~km} / \mathrm{s}$ final speed, such as missions going out of the heliosphere and fast and cheap flyby missions of any target in the outer solar system. Secondly, by inclining the sail to some angle it can also be used to spiral inward in the solar system to study e.g. Mercury and Sun. Also a nonzero inclination with respect to the ecliptic plane is possible to achieve which may be beneficial for observing the Sun. Also the return trip back to Earth from the inner solar system is possible, as is cruising back and forth in the inner solar system and visiting multiple targets such as asteroids. Thirdly, the electric sail could be used to implement a solar wind monitoring spacecraft which is placed permanently between Earth and Sun at somewhere else than the Lagrange point, thus providing a space weather service with more than one hour of warning time. Propulsion and data taking phases probably must be interleaved because ion measurements are not possible when the platform is charged to high positive voltage, although the plasma density and dynamic pressure of the solar wind can probably be sensed by an electron detector and accelerometer even when the electric sail voltage is turned on.

Once accelerated to a high outward speed an electric sailing spacecraft cannot by itself stop to orbit a remote target because the radial component of the thrust is always positive. For stopping under those circumstances one has to use aerocapture or some other traditional technique. Although the electric sail does not provide a marked speed benefit for such missions, being propellantless it might still provide cost saving; this remains to be studied.

In interstellar space the plasma flow is rather slow. Thus the electric sail cannot be used for acceleration, but it can instead be used for braking the spacecraft. There are some concepts such as the laser or microwave sail which are designed to "shoot" a small probe at ultrahigh speed towards e.g. a remote solar system. In these concepts the power source is at Earth so that the accelerated probe needs no propulsive energy source. Stopping the probe at the remote target is very difficult, however, if one has to rely on power beamed from the starting point. The electric sail might then provide a feasible stopping mechanism for such mission concepts. In other words, one would shoot a probe to another solar system at ultrahigh speed using a massive and powerful laser or microwave source installed in near-Earth space, brake the probe before the target by the electric sail action in the interstellar plasma and finally explore the extrasolar planetary 
system with the help of the electric sail and the stellar wind. A similar idea was proposed by Zubrin and Andrews (1990) for their magnetic version of the solar wind sail.

\section{Conclusions}

We have verified using different particle simulations that the theory presented in Sect. 2, in particular Eq. (8), is very likely to be a good approximation to the true force exerted by the solar wind to a thin charged wire. The main independent verifications were the following: (A) The potential expression (2) is in very good agreement with 1-D simulations where there is no solar wind flow. (B) The 2-D simulations are in rather good agreement with the force formula (8).

The electron current gathered by the wire or wire system is likely to be well approximated by the OML theory result, Eq. (9), for reasons explained at the end of Sect. 2.1.

Although the technical and engineering details of how to design a working electric sail are outside the scope of this paper, it is our belief that these issues can be resolved without a need to extrapolate on the current level of technology.

Considering the performance estimates of Sect. 6, we think that it is no exaggeration to say that the electric sail is an extremely promising new propulsion technique whose application area includes (but is not limited to) scientific missions with small to medium payloads where unprecedentedly high final speeds $40-100 \mathrm{~km} / \mathrm{s}$ are required to accomplish the objectives. As a further bonus, if electron heating turns out to be successful it may increase the performance even further.

\section{Appendix A}

\section{Effective electric radius}

Here we consider how to calculate the effective electric radius of a multicomponent wire. Consider $N$ wires whose all mutual distances are equal to $h$. The wires are in vacuum and kept in potential $V_{0}$ with the requirement that the total potential vanishes at $r=r_{0}$ where $r_{0} \gg h \gg r_{w}$ and $r_{w}$ is the single wire radius. The case $N=2$ corresponds to a double wire and $N=3$ corresponds to a triple wire. In the latter case the wires form an equilateral triangle in the perpendicular plane. The vacuum assumption can be made because $h \ll r_{0}$.

In vacuum the potential of a single wire is

$V(r)=V_{0} \frac{\ln \left(r_{0} / r\right)}{\ln \left(r_{0} / r_{w}\right)}$.

The corresponding electric field is

$E(r)=-V^{\prime}(r)=\frac{V_{0} / r}{\ln \left(r_{0} / r_{w}\right)}$.

At the wire surface the electric field is

$E_{0}=E\left(r_{w}\right)=\frac{V_{0} / r_{w}}{\ln \left(r_{0} / r_{w}\right)}$.
Application of Gauß' law gives the line charge $\lambda$ as

$\lambda=\left(\frac{2 \pi \epsilon_{0}}{\ln \left(r_{0} / r_{w}\right)}\right) V_{0}$.

This equation serves as the definition of the effective wire width: if a multiple wire system has total line charge $\lambda$ while being in potential $V_{0}$, its effective width $r_{w}^{*}$ is by definition

$r_{w}^{*}=r_{0} \exp \left(\frac{-2 \pi \epsilon_{0} V_{0}}{\lambda}\right)$.

If the total line charge of the wire system is $\lambda$, due to symmetry each subwire has line charge $\lambda / N$. The potential at the surface of a subwire is then

$V_{0}=\frac{\lambda}{2 \pi \epsilon_{0}}\left[\frac{1}{N} \ln \left(\frac{r_{0}}{r_{w}}\right)+\frac{(N-1)}{N} \ln \left(\frac{r_{0}}{h}\right)\right]$.

Here we used Eq. (A4). The first term in Eq. (A6) is due to the subwire itself and the second term is the potential caused by the other subwires which are at distance $h$. Substituting Eq. (A6) in Eq. (A5) we obtain after simplification

$r_{w}^{*}=r_{w}^{1 / N} h^{1-1 / N}$.

For double wire $(N=2)$ we thus have $r_{w}^{*}=\sqrt{r_{w} h}$ (geometric average $)$ and for triple wire $(N=3)$ we obtain $r_{w}^{*}=\left(r_{w} h^{2}\right)^{1 / 3}$. For example if $r_{w}=10 \mu \mathrm{m}$ and $h=1 \mathrm{~cm}$, the double wire effective radius is $r_{w}^{*}=0.3 \mathrm{~mm}$.

\section{Appendix B}

\section{Electric current gathered by single wire}

In this appendix we derive Eq. (9). Consider a wire of radius $r_{w}$ which resides in potential $V_{0}$ and an incident cold electron beam with density $n$ and speed $v_{0}$. Assume the potential pattern around the wire is cylindrically symmetric so that the attractive Coulomb force is a central force and so both the total energy and angular momentum of incoming electrons are conserved. Assume also that $v_{0} \ll v_{\max }$ where $v_{\max }=\sqrt{2 e V_{0} / m_{e}}$ is the speed of those electrons that nearly touch the wire. Let the incoming electron arrive with impact parameter $y=b$ with $x$-directed velocity (Fig. B1). Then the limiting case when the electron just barely collides with the wire is reached when its velocity at the time of closest approach is equal to $v_{\max }$ in magnitude. At that time the electron's orbit is tangential to the wire and thus its angular momentum $L$ is $L=m_{e} v_{\max } r_{w}$, which must be equal to the original angular momentum $m_{e} v_{0} b$ due to conservation of $L$. Thus we obtain

$b_{\text {limit }}=r_{w}\left(\frac{v_{\max }}{v_{0}}\right)$. 


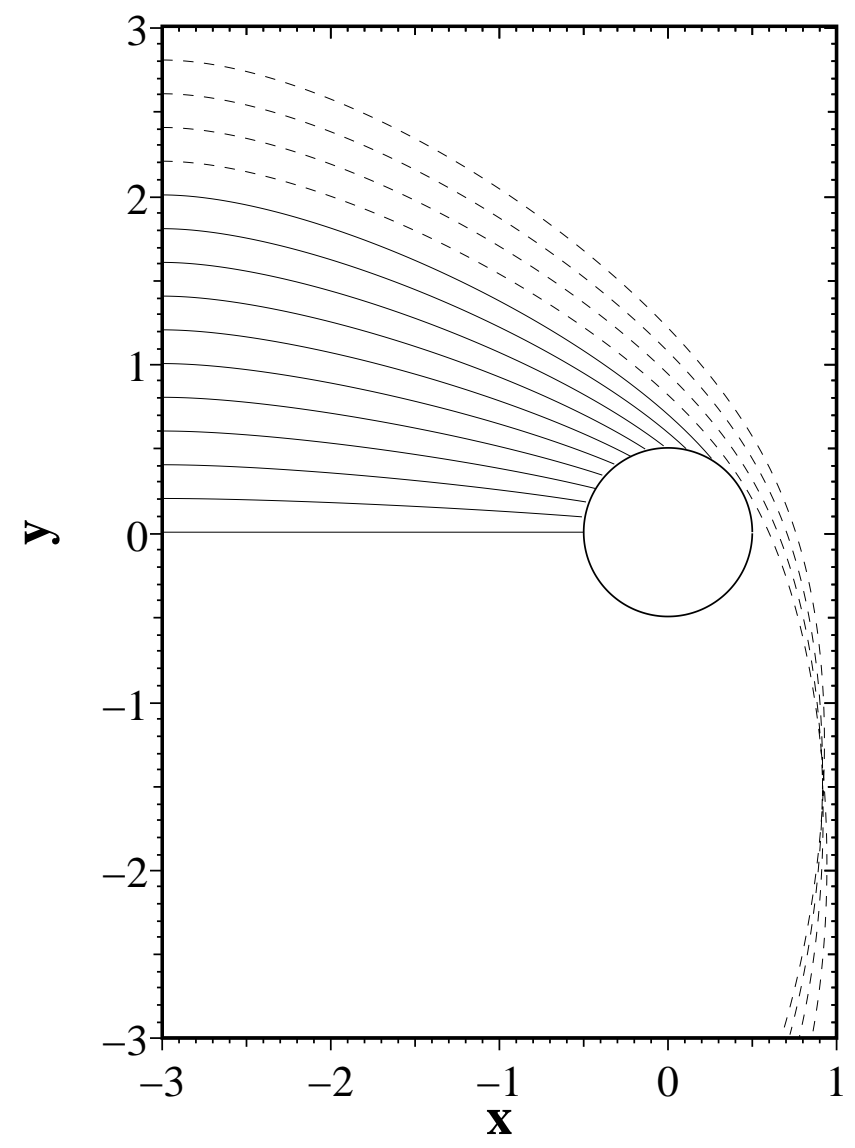

Fig. B1. Geometry of Appendix B. Electrons arrive from the left. Those with small enough impact parameter (initial y-coordinate) hit the wire (solid lines) while others pass by it (dashed lines). The scales are arbitrary.

An electron collides with the wire if and only if the absolute value of its impact parameter is less than $b_{\text {limit. Thus the }}$ electron current gathered by the wire per unit length is

$\frac{d I}{d z}=\left(e n v_{0}\right)\left(2 b_{\text {limit }}\right)=e n v_{\max } 2 r_{w}=e n \sqrt{\frac{e V_{0}}{m_{e}}} 2 r_{w}$

which is Eq. (9). The result depends only on the beam density $n$ but not on its speed $v_{0}$ or incoming direction. Therefore it holds for any electron velocity distribution as long as all the speeds are much lower than $v_{\max }$ because such a distribution can be thought to be composed of a large number of small-density beams with different incoming velocities.

\section{Appendix C}

\section{Solving spacecraft trajectory}

Consider a spacecraft starting from circular solar orbit at $R_{0}=1 \mathrm{AU}$. Its equation of motion is

$\ddot{r}=a-g+\frac{L^{2}}{r^{3}}=a_{0}\left(\frac{R_{0}}{r}\right)^{\alpha}-g_{0}\left(\frac{R_{0}}{r}\right)^{2}+g_{0}\left(\frac{R_{0}}{r}\right)^{3}$

where $a_{0}$ is the sail acceleration at $R_{0}, g_{0}=0.00593 \mathrm{~m} / \mathrm{s}^{2}$ is Sun's gravity field at $R_{0}$ and the exponent $\alpha=7 / 6$ (Eq. 14). Multiplying by $\dot{r}$ we obtain

$\ddot{r} \ddot{r}=\frac{d}{d t}\left(\frac{1}{2} \dot{r}^{2}\right)=a_{0} R_{0}^{\alpha} r^{-\alpha} \dot{r}-g_{0} R_{0}^{2} \frac{\dot{r}}{r^{2}}+g_{0} R_{0}^{3} \frac{\dot{r}}{r^{3}}$.

Integrating with respect to time $t$ from 0 to $t$ we arrive at

$$
\begin{aligned}
\frac{1}{2} \dot{r}^{2} & =a_{0} R_{0}^{\alpha}\left(\frac{r^{1-\alpha}}{1-\alpha}-\frac{R_{0}^{1-\alpha}}{1-\alpha}\right) \\
& -g_{0} R_{0}^{2}\left(\frac{1}{R_{0}}-\frac{1}{r}\right)+\frac{1}{2} g_{0} R_{0}^{3}\left(\frac{1}{R_{0}^{2}}-\frac{1}{r^{2}}\right)
\end{aligned}
$$

For $r \gg R_{0}$ the negative powers of $r$ vanish so we are left with

$\frac{1}{2} \dot{r}^{2}=\frac{a_{0} R_{0}}{\alpha-1}-\frac{1}{2} g_{0} R_{0}$

which can be rearranged to give

$\dot{r}=v_{f}=\sqrt{\left(\frac{2 a_{0}}{\alpha-1}-g_{0}\right) R_{0}}=\sqrt{\left(12 a_{0}-g_{0}\right) R_{0}}$

which is Eq. (15).

Acknowledgements. The authors would like to thank R. Vainio for useful discussions and for comments that have improved the manuscript. R. Hoyt, M. Zavyalov and V. Linkin provided useful technical information. Discussions with E. Keski-Vakkuri, H. Koskinen, H. Laakso, K. Nordlund, J. Polkko, W. Schmidt, J. Silén, M. Uspensky and A. Viljanen are also gratefully acknowledged. The work of A. Sandroos was partly supported by grants from the Academy of Finland and the electric sail work by the Foundation for Finnish Inventions and the Väisälä Foundation.

Topical Editor I. A. Daglis thanks two referees for their help in evaluating this paper.

\section{References}

Allen, J. E.: Probe theory - the orbital motion approach, Physica Scripta, 45, 497-503, 1992.

Birdsall, C. K. and Langdon, A. B.: Plasma physics via computer simulation, Adam Hilger, New York, 1991.

Hoyt, R. and Forward, R. L.: Alternate interconnection Hoytether failure resistant multiline tether, US Pat. 6286788 B1, 2001.

Kivelson, M. G. and Russell, C. T. (Eds.): Introduction to space physics, Cambridge, 1995. 
Janhunen, P., Olsson, A., Vaivads, A., and Peterson, W. K.: Generation of Bernstein waves by ion shell distributions in the auroral region, Ann. Geophys., 21, 881-891, 2003, http://www.ann-geophys.net/21/881/2003/.

Janhunen, P.: Electric sail for spacecraft propulsion, Journal of Propulsion and Power, 20(4), 763-764, 2004.

Mott-Smith, H. M. and Langmuir, I.: The theory of collectors in gaseous discharges, Phys. Rev., 28, 727-763, 1926.

Sittler, E. C. and Scudder, J. D.: An empirical polytrope law for solar wind thermal electrons between 0.45 and $4.76 \mathrm{AU}$ : Voyager 2 and Mariner 10, J. Geophys. Res., 85, 5131-5137, 1980.
Slavin, J. A. and Holzer, R. E.: Solar wind flow about the terrestrial planets, I. Modeling bow shock position and shape, J. Geophys. Res., 86, 11 401-11418, 1981.

Verboncoeur, J. P., Langdon, A. B., and Gladd, N. T.: An objectoriented electromagnetic PIC code, Comput. Phys. Commun. 87, 199-211, 1995.

Zubrin, R. M. and Andrews, D. G.: Magnetic sails and interstellar travel, British Interplanetary Society Journal, 43, 265-272, 1990.

Zubrin, R. M. and Andrews, D. G.: Magnetic sails and interplanetary travel, Journal of Spacecraft and Rockets, 28, 197-203, 1991. 